\title{
A de novo sSMC(22) Characterized by High-Resolution Arrays in a Girl with Cat-Eye Syndrome without Coloboma
}

\author{
C. Córdova-Fletes ${ }^{a, b}$ M.G. Domínguez ${ }^{c, d}$ A. Vázquez-Cárdenas ${ }^{e} \quad$ L.E. Figuerac, d
}

\author{
V.A. Neira ${ }^{d} \quad$ A. Rojas-Martínez ${ }^{\text {a,b }}$ R. Ortiz-López ${ }^{a, b}$ \\ aDepartamento de Bioquímica y Medicina Molecular, Facultad de Medicina, Universidad Autónoma de Nuevo León, \\ bunidad de Biología Molecular, Genómica y Secuenciación, Centro de Investigación y Desarrollo en Ciencias \\ de la Salud, Universidad Autónoma de Nuevo León, Monterrey, ' División de Genética, Centro de Investigación \\ Biomédica de Occidente, CMNO-IMSS, dCentro Universitario de Ciencias de la Salud, Universidad de Guadalajara, \\ eDepartamento de Genética, Instituto de Ciencias Biológicas, Universidad Autónoma de Guadalajara, \\ Guadalajara, México
}

\section{Key Words}

Cat-eye syndrome - Microarrays - Phenotypic variability • 22q supernumerary marker

\begin{abstract}
Cat-eye syndrome (CES) results from trisomy or tetrasomy of proximal 22q originated by a small supernumerary marker chromosome (sSMC). Two critical regions for the major clinical features of CES (CESCRs) have been suggested; however, CES clinical presentation often does not correlate with the sSMC genetic content. We report here a CES girl without coloboma and carrier of a de novo type I sSMC(22) as determined by G- and C-banding, NOR staining and microarrays. This sSMC included 6 distal genes outside the original CESCR and led to a tetrasomy for 22q11.1-22q11.21. The patient's final karyotype was 47,XX,+psu dic(22)(q11.21).arr 22q11. $1 q 11.21(15,250,000-17,035,860) \times 4 \mathrm{dn}$. The amplified region outside of CESCR included some genes that may be related to neurologic, heart and renal abnormalities. Conversely, even though the amplification included the CECR2 gene, a major candidate for eye features, there was no coloboma in
\end{abstract}

the patient. The genetic delineation of the present sSMC further strengthens that the CES clinical presentation does not fit completely with the duplicated genetic content and that CES is actually a genomic disorder. Furthermore, since we observed no mosaicism, we believe that other mechanisms might be behind the variability of CES phenotypes as well, mainly those related with functional interactions among amplified genes.

Copyright $\odot 2012$ S. Karger AG, Basel

The Schmid-Fraccaro or cat-eye syndrome (CES) is characterized by unilateral or bilateral ocular coloboma, downslanted palpebral fissures, pre-auricular pits or tags, heart and renal malformations, anal fistula and/or atresia, and neuropsychomotor disability [Rosias et al., 2001; Romagna et al., 2010; OMIM 115470]. The fact that the phenotype is highly variable is illustrated by the coloboma being present in only $\sim 50 \%$ of patients [Jezela-Stanek et al., 2009]. The CES results from a partial trisomy or tetrasomy of proximal 22q due to a small supernumerary marker chromosome (sSMC) [Mears et al., 1994; Mark et

\section{KARGER}

Fax +41613061234

E-Mail karger@karger.ch

www.karger.com (c) 2012 S. Karger AG, Basel

$1661-8769 / 12 / 0033-0131 \$ 38.00 / 0$

Accessible online at:

www.karger.com/msy
Carlos Córdova-Fletes, Unidad de Biología Molecular, Genómica y Secuenciación Centro de Investigación y Desarrollo en Ciencias de la Salud Universidad Autónoma de Nuevo León

Calle Dr. Carlos Canseco s/n. Colonia Mitras Centro, Monterrey, AP 64460 (Mexico) Tel. +52 811340 4370, E-Mail carlos.cordovafl@uanl.edu.mx 


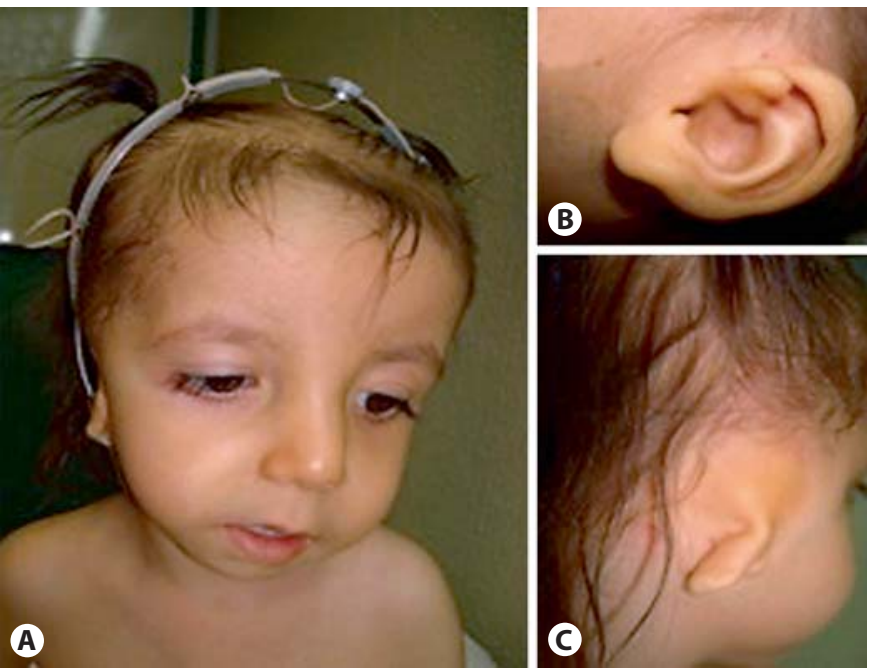

Fig. 1. A Craniofacial features of the patient. B Left pre-auricular tag. C Dysplastic right ear.

al., 2005; Bélien et al., 2008; Jezela-Stanek et al., 2009], and less commonly from an interstitial duplication of the CES critical region (CESCR) [Meins et al., 2003]. The original CESCR spans around $2 \mathrm{Mb}$, from the centromere to the D22S57 marker [Mears et al., 1994], and includes the CECR1 and CECR2 genes as major candidates for heart/facial and neurologic/eye features, respectively [Riazi et al., 2000; Banting et al., 2005]. In the CES, 3 sSMC types (I, IIa-b and III) related to low copy repeats in 22q11.2 (LCR22s) have been described [Bartsch et al., 2005a; Bélien et al., 2008]. We report here a CES girl without coloboma but carrying a de novo type I sSMC(22) in order to provide further insights about CES phenotypic variability.

\section{Clinical Report and Methods}

\section{Patient Data}

The girl is the 3rd child of a G4P3A1 mother and an unrelated father. Prenatal ultrasonography disclosed right microtia, anal atresia, small dysplastic right kidney, and oligohydramnios. At birth, she was diagnosed with patent ductus arteriosus, which closed spontaneously. Her craniofacial features (fig. 1) included broad and prominent forehead, sparse hair, downslanting palpebral fissures, bilateral ptosis of the eyelids, telecanthus, strabismus, facial asymmetry, right microtia, left pre-auricular tag, long philtrum, thin upper lip, and micro-/retrognathia; breast hypoplasia was detected, too. Noticeably, a detailed ophthalmologic inspection did not show any eye coloboma. She underwent surgical treatment for the anal atresia and rectovaginal fistula. On ex- amination at 2 years, her weight $(8,000 \mathrm{~g})$ and height $(77 \mathrm{~cm})$ were below the 3 rd centile. She also presented delayed psychomotor development.

\section{Cytogenetic Analyses}

The initial cytogenetic analyses of the patient, her parents and a sister with cleft lip and palate were made on G-banded chromosomes obtained from 72-hour lymphocyte cultures. To confirm the presence of 2 centromeres and satellites in the sSMC, C-banding and NOR staining were realized.

\section{Array Comparative Genomic Hybridization}

To ascertain the chromosomal origin and segmental composition of the sSMC, a high-resolution genomic scan using Affymetrix GenomeWide SNP 6.0 platform was performed in the patient and her parents. Analysis of the arrays was performed using Genotyping Console v4.0 and ChAS v1.2 software. All samples were taken after an informed consent had been signed.

\section{Results}

The patient's G-banded karyotype was $47, \mathrm{XX},+$ mar [30]; karyotypes of the patient's relatives were normal. Cbanding and NOR staining confirmed that the stable sSMC was bisatellited and pseudodicentric (fig. 2). In addition to identifying the origin of the marker to be chromosome 22 , the microarray assay disclosed an amplified 2.6- $\mathrm{Mb}$ region (genomic position 15,250,000-17,035,860) embracing 1,177 markers (NCBI36/hg18) and entailing a partial tetrasomy for 22q11.1-22q11.21 (fig. 2). There were 4 copies of XKR3, GAB4, CECR7, IL17RA, CECR6, CECR5, CECR1 (at 22q11.1), CECR2, SLC25A18, ATP6V1E1, MIL1 (also named BCL2L13), BID, MICAL3, PEX26, TUBA8 and USP18 (at 22q11.21) genes. The amplification did not overlap with the VCF/DiGeorge locus. Thus, the sSMC was classified as type I with breakpoints within LCR223a and flanked by D22S427 and D22S36 [McTaggart et al., 1998; Bartsch et al., 2005a]. Microarray results from the parents were normal. The final karyotype based on ISCN [2009] was 47,XX,+psu $\operatorname{dic}(22)(\mathrm{q} 11.21)$.arr 22q11. $1 \mathrm{q} 11.21(15,250,000-17,035,860) \times 4 \mathrm{dn}$. The present approach, however, was not sufficient to define the parental chromosomal origin of the sSMC.

\section{Discussion}

Here, we thoroughly characterized a constitutional de novo type 1 sSMC(22) with both breakpoints within the LCR22-3a interval but including 6 genes outside the original CESCR (fig. 2). 


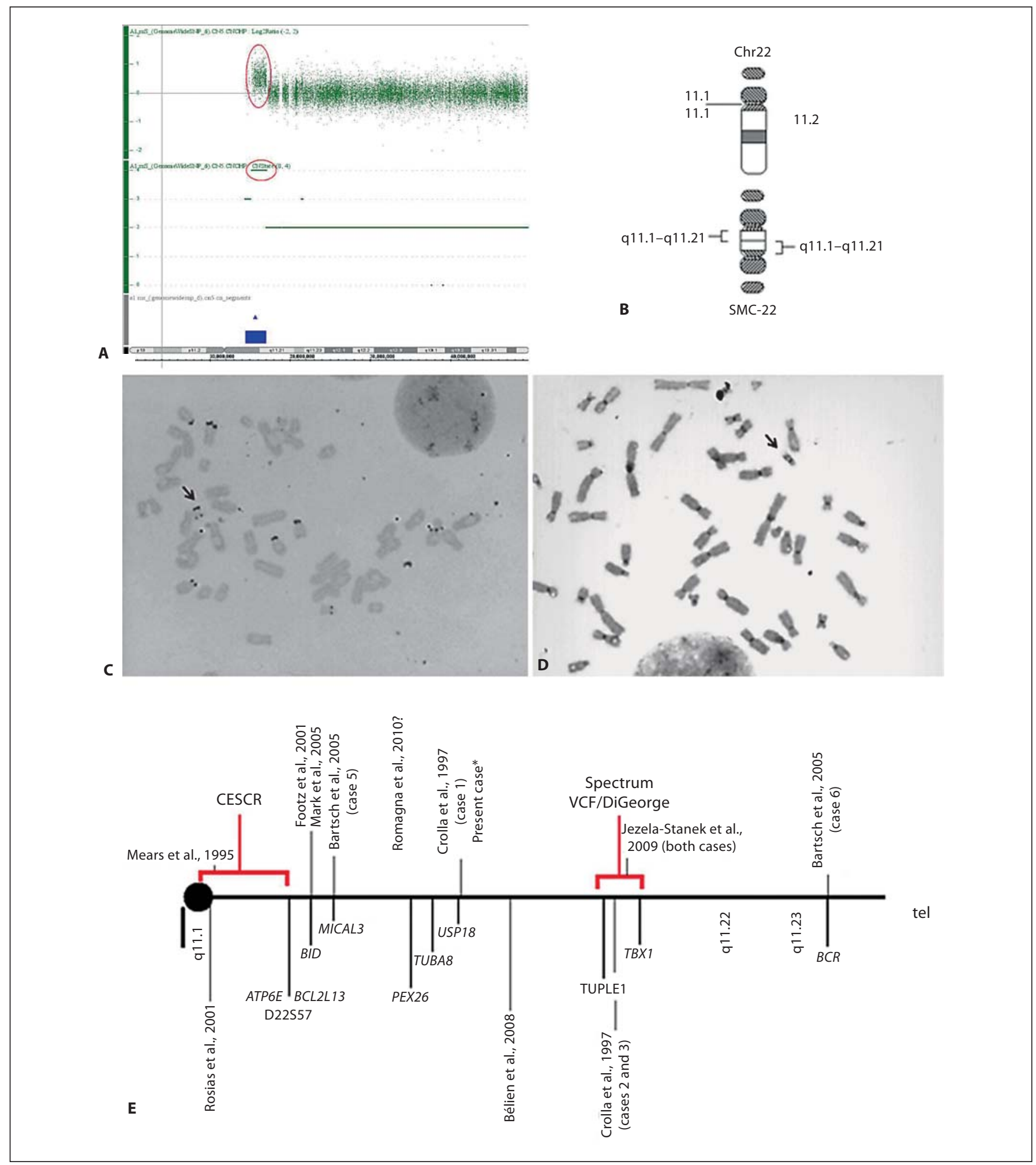

Fig. 2. A Gain profile analysis indicated by $\log _{2}$ ratio (circled plotting) and copy number state (circled bars) visualization. Blue bar represents gains. B Ideograms from normal chromosome 22 and sSMC(22). C and D Partial metaphases showing the sSMC; C NOR staining shows a bisatellited marker (arrow), and D C-banding denoting a pseudodicentric marker (arrow). E Diagram (not to scale) denoting CESCR and beyond as well as the amplified interval reported here. Black lines along with references indicate the reported breakpoint for each amplified interval in that case. 
Breakpoints observed place USP18 as the more distal gene (within D22S427), and suggest that formation of the present sSMC(22) could have resulted from a double rupture within LCR22-3a and inter- or intrachromosomal 'U-type' reunion during meiosis I, in accordance to other sSMCs [Van Dyke et al., 1977; Wandstrat and Schwartz, 2000; Murmann et al., 2009].

The amplified gene content in the present case agrees with most of CES cardinal clinical features, except for coloboma that was absent in spite of CECR 2 overdosage. This fact suggests that other unknown factors beside CECR2 amplification are required to generate a coloboma. In line, a $600-\mathrm{kb}$ intrachromosomal triplication spanning only CECR2, SLC25A18 and ATP6V1E1 genes was described in patients with 3 CES traits but neither coloboma nor mental disability [Knijnenburg et al., 2012].

Currently, 2 CESCRs that altogether extend beyond the D22S57 marker and include BID as most distal gene, have been suggested to account for most CES clinical features including mental disorders [Mears et al., 1994; Footz et al., 2001]. In addition, other CES cases with larger amplified regions (even encompassing VCF/DiGeorge locus or further) have been described (fig. 2) [Crolla et al., 1997; Bartsch et al., 2005a; Mark et al., 2005; Bélien et al., 2008; Jezela-Stanek et al., 2009]. Despite these observations, there is no precise correlation between the genetic content of the $\operatorname{sSMC}(22) s$ and clinical features [Crolla et al., 1997; Rosias et al., 2001; Bartsch et al., 2005a; JezelaStanek et al., 2009]. This fact may be due, among other factors, to mosaicism for sSMC(22)s [Bartsch et al., 2005a, b]. Furthermore, a refined delineation of the $\operatorname{sSMC}(22) \mathrm{s}$ is often lacking; e.g. just $\sim 11 / 200$ CES cases have been delineated by more sensible techniques as microarrays [Liehr, 2012].

The description of at least 2 CESCRs and multiple amplified intervals within 22q coupled to CES phenotypic variants further strengthens that CES is a genomic disorder or contiguous gene syndrome [McDermid and Morrow, 2002]. Thereby, CES clinical spectrum could partially be due to overdosage of genes outside one or both
CESCRs. Accordingly, the gene content of the present sSMC(22) included, in addition to both CESCRs, 3 distal genes whose overdosage (along with that of MIL1 and BID, suggested by Footz et al. [2001]) could mainly be related to the neurologic impairment in this and other CES patients: MICAL3 and TUBA 8 are implicated in neuronal migration [Bron et al., 2007; Abdollahi et al., 2009] while PEX26 is highly expressed in kidneys, brain and heart (UCSC genome browser).

However, since this assertion does not perfectly match, we speculate that positive or negative interactions among amplified genes could represent an alternative mechanism to account for the variability of CES phenotypes. This notion is supported by the observation that MIL1 and BID have opposite functions within the same pathway (inhibition and activation, respectively) and that a probable antisense regulation among them was suggested [Kanehisa and Goto, 2000; Footz et al., 2001]. In addition, an analysis of the protein-protein interaction network for proximally or distally amplified genes in CES exhibited, among many others, functional interactions of the MIL1 and BID genes within the same cluster (see online suppl. figs. 1 and 2, www.karger.com/doi/10.1159/000341632).

Finally, we consider it helpful to finely characterize those cases with only cardinal clinical traits of CES in order to complete the list of genes related to the major features of the disease as well as to perform a functional analysis of the amplified gene content. These approaches will ultimately result in a better disease understanding and classification.

\section{Acknowledgements}

We thank the patient's parents for their support for this research. This work was partially supported by PROMEP (No. 103.5/11/4330) for C.C.-F., PAICYT (No. SA324-10) and FOMIX (convocatoria M0014-2007-2010, Reg. 068251) for CIDICS. V.A.N. is supported by CONACyT and SENESCYT scholarships. We thank Dr. H. Rivera for his support in reviewing our study.
References modeling complex with SNF2L. Hum Mol Genet 14:513-524 (2005) Hayward BE, et al: Mutation of the variant alpha-tubulin TUBA8 results in polymicrogyria with optic nerve hypoplasia. Am J Hum Genet 85:737-744 (2009).

Banting GS, Barak O, Ames TM, Burnham AC, Kardel MD, et al: CECR2, a protein involved in neurulation, forms a novel chromatin re-
Bartsch O, Rasi S, Hoffmann K, Blin N: FISH of supernumerary marker chromosomes (SMCs) identifies six diagnostically relevant intervals on chromosome $22 \mathrm{q}$ and a novel type of bisatellited SMC(22). Eur J Hum Genet 13:592-598 (2005a). 
Bartsch O, Loitzsch A, Kozlowski P, Mazauric ML, Hickmann G: Forty-two supernumerary marker chromosomes (SMCs) in 43,273 prenatal samples: chromosomal distribution, clinical findings, and UPD studies. Eur J Hum Genet 13:1192-1204 (2005b).

-Bélien V, Gérard-Blanluet M, Serero S, Le Dû N, Baumann C, et al: Partial trisomy of chromosome 22 resulting from a supernumerary marker chromosome 22 in a child with features of cat eye syndrome. Am J Med Genet A 146A:1871-1874 (2008).

Bron R, Vermeren M, Kokot N, Andrews W, Little GE, et al: Boundary cap cells constrain spinal motor neuron somal migration at motor exit points by a semaphorin-plexin mechanism. Neural Dev 2:21 (2007).

-Crolla JA, Howard P, Mitchell C, Long FL, Dennis NR: A molecular and FISH approach to determining karyotype and phenotype correlations in six patients with supernumerary marker(22) chromosomes. Am J Med Genet 72:440-447 (1997).

Footz TK, Brinkman-Mills P, Banting GS, Maier SA, Riazi MA, et al: Analysis of the cat eye syndrome critical region in humans and the region of conserved synteny in mice: a search for candidate genes at or near the human chromosome 22 pericentromere. Genome Res 11:1053-1070 (2001).

ISCN 2009: An International System for Human Cytogenetic Nomenclature. Shaffer LG, Slovak ML, Campbell LJ (eds) (S. Karger, Basel 2009).

Jezela-Stanek A, Dobrzańska A, Maksym-Gasiorek D, Trzeciakowski W, Gutkowska A, et al: Trisomy 22pter-q12.3 presenting with hepatic dysfunction variability of cat-eye syndrome. Clin Dysmorphol 18:13-17 (2009).
Kanehisa M, Goto S: KEGG: Kyoto Encyclopedia of Genes and Genomes. Nucleic Acids Research 28:27-30 (2000).

Knijnenburg J, van Bever Y, Hulsman LO, van Kempen CA, Bolman GM, et al: A $600 \mathrm{~kb}$ triplication in the cat eye syndrome critical region causes anorectal, renal and preauricular anomalies in a three-generation family. Eur J Hum Genet (2012), E-pub ahead of print.

Liehr T: Small supernumerary marker chromosomes. 2012. http://www.fish.uniklinikumjena.de/sSMC/sSMC+by+chromosome/ sSMC+22.html (accessed May 30, 2012).

- Mark HF, Wyandt H, Huang XL, Milunsky JM: Delineation of a supernumerary marker chromosome utilizing a multimodal approach of G-banding, fluorescent in situ hybridization, confirmatory P1 artificial chromosome fluorescent in situ hybridization, and high-resolution comparative genomic hybridization. Clin Genet 68:146-151 (2005).

McDermid HE, Morrow BE: Genomic disorders on 22q11. Am J Hum Genet 70:1077-1088 (2002).

McTaggart KE, Budarf ML, Driscoll DA, Emanuel BS, Ferreira P, et al: Cat eye syndrome chromosome breakpoint clustering: identification of two intervals also associated with 22q11 deletion syndrome breakpoints. Cytogenet Cell Genet 81:222-228 (1998).
- Mears AJ, Duncan AM, Budarf ML, McDermid HE, Patil SR: Molecular characterization of the marker chromosome associated with cat eye syndrome. Am J Hum Genet 55:134-142 (1994).

Meins M, Burfeind P, Motsch S, Trappe R, Bartmus D, et al: Partial trisomy of chromosome 22 resulting from an interstitial duplication of 22q11.2 in a child with typical cat eye syndrome. J Med Genet 40:e62 (2003).

- Murmann AE, Conrad DF, Mashek H, Curtis CA, Nicolae RI, et al: Inverted duplications on acentric markers: mechanism of formation. Hum Mol Genet 18:2241-2256 (2009).

Riazi MA, Brinkman-Mills P, Nguyen T, Pan H, Phan S, et al: The human homolog of insectderived growth factor, CECR1, is a candidate gene for features of cat eye syndrome. Genomics 64:277-285 (2000).

- Romagna ES, Appel da Silva MC, Ballardin PA Schmid-Fraccaro syndrome: severe neurologic features. Pediatr Neurol 42:151-153 (2010).

-Rosias PR, Sijstermans JM, Theunissen PM, Pulles-Heintzberger CF, De Die-Smulders $\mathrm{CE}$, et al: Phenotypic variability of the cat eye syndrome. Case report and review of the literature. Genet Couns 12:273-282 (2001).

-Van Dyke DL, Weiss L, Logan M, Pai GS: The origin and behavior of two isodicentric bisatellited chromosomes. Am J Hum Genet 29: 294-300 (1977).

-Wandstrat AE, Schwartz S: Isolation and molecular analysis of inv dup(15) and construction of a physical map of a common breakpoint in order to elucidate their mechanism of formation. Chromosoma 109:498-505 (2000). 